\title{
Association of constitutively activated hepatocyte growth factor receptor (Met) with resistance to a dual EGFR/Her2 inhibitor in non-small-cell lung cancer cells
}

\author{
S Agarwal ${ }^{*, 1,2}$, C Zerillo ${ }^{2}$, J Kolmakova ${ }^{1,4}$, JG Christensen ${ }^{3}$, LN Harris ${ }^{2}$, DL Rimm', MP DiGiovanna ${ }^{2,5}$ and \\ DF Stern ${ }^{1,5}$
}

'Department of Pathology, Yale University School of Medicine, New Haven, CT 06520, USA; '2Department of Internal Medicine, Section of Medical Oncology, Yale Comprehensive Cancer Center, Yale University School of Medicine, New Haven, CT 06520, USA; ${ }^{3}$ Department of Research Pharmacology, Pfizer Global Research and Development, La Jolla, CA 92121 , USA

\begin{abstract}
There is a pressing need to identify new drug targets and novel approaches for treatment of non-small-cell lung carcinoma (NSCLC). Members of the epidermal growth factor receptor (EGFR) and Met receptor families have been identified as important molecular targets for NSCLC. Two EGFR tyrosine kinase inhibitors (TKIs; erlotinib and gefitinib) are in current clinical use, but a majority of patients do not respond to these targeted therapies. We used receptor TK (RTK) capture arrays to identify receptors active in NSCLC cell lines. As Met and ErbBs were active, we explored the potential therapeutic advantage of combined targeting of Met with ErbB receptor family inhibitors for treatment of NSCLC. We found that Met physically interacts with both EGFR and Her2 in a NSCLC cell line with overexpression/overactivation of Met. Combined use of a dual EGFR/Her2 inhibitor with a Met inhibitor yields maximal growth inhibition compared with the use of EGFR and/or Met inhibitors. This suggests that simultaneous inhibition of multiple RTKs may be needed to effectively abrogate tumour cell growth. Phosphoproteomic analysis by RTK capture arrays may be a valuable tool for identifying the subset of tumours with functional receptor activation, regardless of mechanism.

British Journal of Cancer (2009) I 00, 94I-949. doi:I0.1038/sj.bjc.6604937 www.bjcancer.com
\end{abstract}

Published online 24 February 2009

(c) 2009 Cancer Research UK

Keywords: Met; Her/ErbB receptor family; lung cancer; signal transduction; RTK

Non-small-cell lung cancer (NSCLC) accounts for the majority of all lung cancer cases and, with poor prognosis and low cure rate, is a leading cause of cancer mortality. Tyrosine kinase inhibitors (TKIs) have emerged as an important class of drugs for the treatment of NSCLC. Gefitinib (Iressa) and erlotinib (Tarceva) are the two US FDA-approved epidermal growth factor receptor (EGFR)-specific TKIs that have shown clinical benefit in treating NSCLC. They appear to work, in part, by inhibiting the effects of gain-of-function mutations in EGFR, but patients with tumours lacking mutations benefit as well (Sordella et al, 2004; Mukohara et al, 2005). Gain-of-function mutations are clustered around the catalytic domain of EGFR and are either single amino-acid substitutions or small insertions/deletions. However, not all patients carrying activating mutant EGFR benefit from TKI therapy and almost all acquire resistance within a year after initial response (Haber et al, 2005; Riely et al, 2006; Sharma et al, 2007).

A major mechanism of resistance to TKI-based therapy is the development of a second site mutation (T790M) in EGFR causing a

\footnotetext{
*Correspondence: Dr S Agarwal; E-mail: seema.agarwal@yale.edu

${ }^{4}$ Current address: Boston University School of Medicine, Boston, MA, USA

${ }^{5}$ These authors contributed equally to this work

Received I December 2008; revised 14 January 2009; accepted 19 January 2009; published online 24 February 2009
}

conformational change that inhibits effective binding of the kinase inhibitors to the ATP pocket (Liu et al, 2006). Another secondary mutation (D761Y) in EGFR was identified in an NSCLC brain metastasis originating from a primary tumour that initially responded to gefitinib-based therapy (Balak et al, 2006). Another route for escape from TKI therapy is the acquired amplification of the hepatocyte growth factor (HGF) receptor, MET, which was found in four of eighteen (22\%) resistant NSCLC (Engelman et al, 2007). The majority of the amplifications were found in metastatic lesions, suggesting that Met may be involved in the development of metastases as well as acquired resistance to TKI therapy.

Met plays a critical role in cancer, liver and kidney regeneration and mammary gland development, including cell proliferation, motility, invasion and branching tubulogenesis (Yang et al, 1995; Rosario and Birchmeier, 2003; Gao and Vande Woude, 2005; Gao et al, 2005; Desiderio, 2007; Sattler and Salgia, 2007). Met is present in all tissue types, and activation of Met by its ligand, HGF, leads to the activation of several signalling pathways that coordinate to achieve Met-dependent cellular functions. In normal cells, Met localises predominantly at the plasma membrane; however, in the germinal tissue layer of normal colon, skin and testis, and in cancerous tissue, both cytoplasmic and nuclear localisation have been observed (Pozner-Moulis et al, 2006). Gain-of-function mutations, overexpression or amplification of MET have been identified and are associated with tumour growth and metastasis (Ma et al, 2003; Lengyel et al, 2005; Kong-Beltran et al, 2006). 
Although a small fraction of NSCLC patients $(\sim 10 \%)$ have major objective responses to EGFR-based therapy, the majority of NSCLC patients do not respond to EGFR-targeted therapies. Thus, there is a pressing clinical need for the identification of new drug targets and new treatment strategies. It is known that EGFR signalling is modulated by other receptor tyrosine kinases (RTKs). For example, it is well established that heterodimerisation with other ErbB family receptors, Her2 and Her3, augments the oncogenic activities of EGFR (Engelman et al, 2005, 2007; Arteaga, 2007). Furthermore, recent evidence implicates Met in functional interactions with EGFR and Her3 (Jo et al, 2000). As both the ErbB family of receptors and Met are promising molecular targets for therapy of NSCLC, and with evidence for functional interactions of these receptors, we have explored the possibility that combined targeting of Met and one or more ErbB family members may have therapeutic promise.

\section{MATERIALS AND METHODS}

\section{Cell lines and other reagents}

H441 and H1666 cells were purchased from ATCC (Manassas, VA, USA) and were maintained in RPMI supplemented with $10 \%$ FBS, sodium pyruvate, glutamine, penicillin and streptomycin in a $37^{\circ} \mathrm{C}$ incubator containing $5 \% \mathrm{CO}_{2} .32 \mathrm{D} / \mathrm{Met}$ cells were generously provided to us by Dr Donald Bottaro from the National Cancer Institute, Bethesda, MD, USA. These cells were maintained in RPMI medium with $10 \%$ WEHI-conditioned medium to provide IL-3 (Day et al, 1999). PHA665752 (a small molecule TKI for Met) was a generous gift from Pfizer (La Jolla, CA, USA), GW2974 (a dual small molecule TKI for both EGFR and Her2) was purchased from Calbiochem (Gibbstown, NJ, USA) and gefitinib (a small molecule TKI for EGFR) was purchased from Biaffin GmbH \& Co KG (Kassel, Germany). All drugs were dissolved in DMSO to produce 20-mM stock solutions. Rabbit anti-EGFR, mouse anti-EGFR, rabbit anti-Met, rabbit anti-Her2, mouse anti-Her3, mouse IgG, goat antimouse HRP and goat antirabbit HRP antibodies were purchased from Santa Cruz Biotechnology (Santa Cruz, CA, USA); mouse anti-Her2 was purchased from Labvision (Fremont, CA, USA); rabbit anti-Her3, rabbit anti-Akt, rabbit antiphospho-Akt, rabbit anti-Erk1/2, rabbit anti-phospho-Erk1/2, mouse antiphosphotyrosine, mouse anti-Stat3, rabbit antiphospho-Stat3 (Ser 727), rabbit antiphospho-Stat3 (Y705), mouse anti-Met, rabbit antiphospho-Met (Y1234/1235), rabbit antiphospho-EGFR (Y1068), rabbit antiphospho-EGFR (Y992), rabbit antiphospho-EGFR (845) and rabbit anti- $\beta$-tubulin were purchased from Cell Signalling Technology (Danvers, MA, USA); rabbit anti-Shc was purchased from Upstate Cell Signalling Solutions (Billerica, MA, USA); and rabbit antiphospho-Shc was purchased from Sigma-Aldrich (St Louis, MO, USA). Epidermal growth factor (EGF), HGF and human phospho-RTK array kits were purchased from R\&D Systems (Minneapolis, MN, USA).

\section{Receptor tyrosine kinase antibody array profile}

Either $200 \mu \mathrm{g}$ (Figures 1A and 5A) or $500 \mu \mathrm{g}$ (Figure 2A) of whole cell extracts were analysed on human phospho-RTK arrays from R\&D Systems according to the manufacturer's recommendation. Details of the protocol are provided in the Supplementary section.

\section{Immunoprecipitation (IP) and immunoblot}

For IP experiments, cells were incubated with either vehicle $(0.5 \%$ DMSO) or with indicated concentrations of GW2974 in $0.5 \%$ DMSO containing cell media for $2 \mathrm{~h}$. Detailed protocols are in the Supplementary section.

\section{Cell proliferation assay}

Cells were plated on 96-well plates in $50 \mu \mathrm{l}$ of media at a density of 3000 cells per well for H441 cells or 10000 cells per well for H1666 cells. Compounds were serially diluted into cell media as $2 \times$ stocks containing $1 \%$ DMSO, and $50 \mu \mathrm{l}$ of each dilution was added to the wells in triplicate. After 5 days of incubation with the compounds, cell survival was assessed by adding $10 \mu \mathrm{l}$ of WST-1 [2-(4iodophenyl)-3-(4-nitrophenyl)-5-(2,4-disulphophenyl)-2H tetrazolium] reagent (Roche Applied Sciences, Indianapolis, IN, USA) in each well of a 96-well plate. After incubation for $3 \mathrm{~h}$, the plates were read in a plate reader (Spectramax 250; Molecular Devices, Sunnyvale, CA, USA) at $450 \mathrm{~nm}$. The zero time for cells with only $0.5 \%$ DMSO was also assessed for each plate and was subtracted from the 5-day time points. The XLfit program (ID Business Solutions, Parsippany, NJ, USA) was used to obtain and calculate the $\mathrm{GI}_{50}$ curves for each compound using a non-linear regression curve fit utilising Lavenburg-Marquardt algorithm. Each experiment was performed a minimum of three times.

\section{Hepatocyte growth factor activation}

Cells were plated in 24-well plate in $750 \mu$ l of starvation media (no FBS and no IL-3 containing WEHI media) at a density of $5 \times 10^{6}$ cells per well for 32D/Met cells. After $2 \mathrm{~h}$ of starvation, compounds were serially diluted into cell starvation media as $2 \times$ stocks containing $1 \% \mathrm{DMSO}$, and $750 \mu \mathrm{l}$ of each dilution was added to the wells. Details of the protocol are in the Supplementary section.

\section{Lentivirus infection for Met shRNA}

Met shRNA cloned in pLKO.1 puro vector (Engelman et al, 2007) and empty pLKO.1 puro vector were used for lentivirus production and infection in H441 cells as described (Engelman et al, 2007). Stably infected cells were selected in $2 \mu \mathrm{g} \mathrm{ml}^{-1}$ of puromycin (Promega, Madison, WI, USA).

\section{RESULTS}

\section{Activation/overexpression of Met and resistance to GW2974}

Activation of multiple RTKs simultaneously can contribute to the malignant phenotype in NSCLC. Thus, we used a RTK antibody array that captures 42 receptors and was then probed with antiphosphotyrosine to survey the activity of RTKs in NSCLC lines H441 and H1666. These lines have wild-type (WT) EGFR. Epidermal growth factor receptor and Her3 were highly activated in H1666 cells, and EGFR, Her2 and Her3 in H441 cells (Figure 1A). Both cell lines showed weak activation of MSPR and DTK, and some Her4 activity was detected in H441 cells (Figure 1A). In addition, H441 cells showed strong activation of Met, consistent with earlier reports (Christensen et al, 2003), whereas activated Met was not detected in H1666 cells (Figure 1A and $\mathrm{C}$ ).

With the potential importance of ErbB receptor inhibitors for lung cancer treatment, and with multiple ErbB receptors activated in these cell lines, we investigated their sensitivity to the dual EGFR/Her2 kinase inhibitor GW2974 in proliferation assays. GW2974 is an analogue of lapatinib with an enzymatic acitivity of $0.007 \mu \mathrm{M}$ (compared with $0.012 \mu \mathrm{M}$ for lapatinib) for EGFR and $0.016 \mu \mathrm{M}$ (compared with $0.010 \mu \mathrm{M}$ for lapatinib) for Her2 (Rusnak et al, 2001; Petrov et al, 2006). Cell growth was assessed after a 5-day continuous exposure to drug. H1666 cells showed high sensitivity to GW2974 with an average $\mathrm{GI}_{50}$ of $0.1 \mu \mathrm{M}$, compared with $\mathrm{H} 441$, where the $\mathrm{GI}_{50}$ was in the micromolar range $(6-9 \mu \mathrm{M}$; Figure 1B).

We examined the effects of GW2974 on signalling pathways both at the receptor level and for downstream signalling components (Figure 1C). GW2974 inhibited EGFR phosphorylation in H441 cells in a dose-dependent manner, although at micromolar concentrations. Surprisingly, phospho-Met was also inhibited 
A

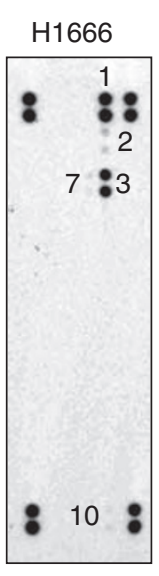

B

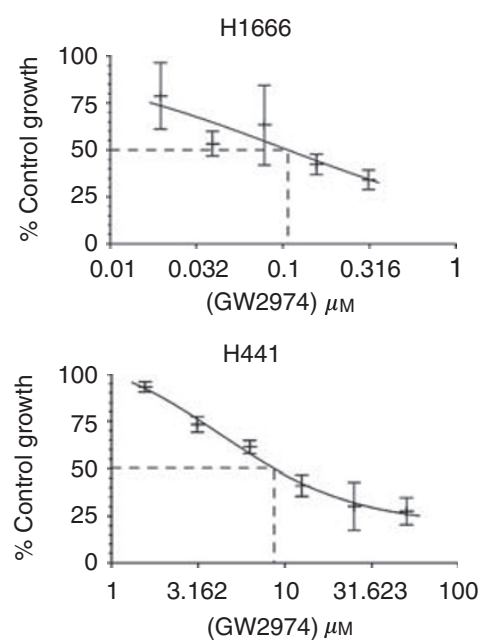

(GW2974) $\mu \mathrm{M}$
C

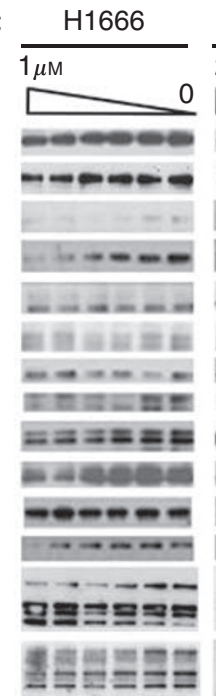

123456

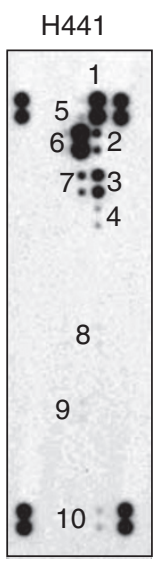

$\mathrm{H} 441$

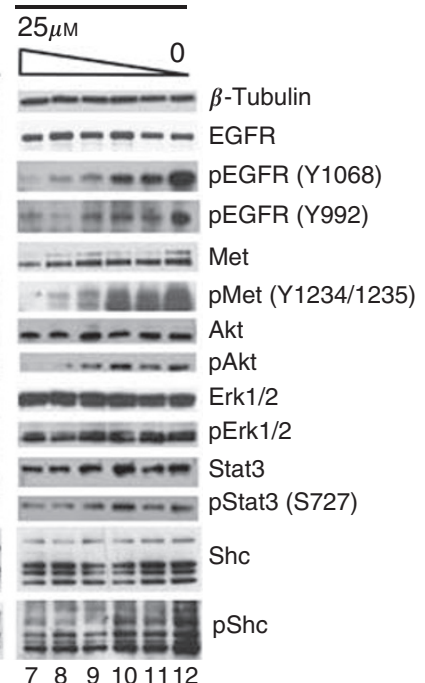

Figure I Activation of Met and response to GW2974 in H44I cells. (A) Multiple RTKs are activated in H44I and HI666 cells in full serum conditions. Whole cell extracts $(200 \mu \mathrm{g})$ were incubated with RTK capture array membranes. RTK activation was determined by probing with phosphotyrosine antibody conjugated to horse-radish peroxidase. Paired spots correspond to I: EGFR; 2: Her2; 3: Her3; 4: Her4; 5: Mer; 6: Met; 7: MSPR; 8: Flt3; 9: Ret; and I0: Dtk. The four sets of duplicate spots at each corner of the RTK array membrane serve to orient and align the membrane to identify and correlate the positive set of spots to individual RTKs. (B) Sensitivity of cell lines to GW2974. Cells were treated with indicated concentrations of GW2974 for 5 days, and cell proliferation was measured using a WST-I colorimetric assay. The GI 50 of HI 666 is $0.1 \mu$ M compared with $8.6 \mu$ M for H44I cells. Error bars represent s.d. of three data sets. Representative data are shown from multiple experiments. (C) Dose-dependent inhibition of EGFR-dependent signalling in HI666 cells and Met-dependent signalling in $\mathrm{H44I}$ cells by GW2974. Whole cell extracts were made from cells treated with GW2974 at indicated concentrations for 5 days as in panel $\mathrm{B}$, and subjected to immunoblotting with the indicated antibodies. Arrows indicate respective protein bands. $\beta$-Tubulin was used as a loading

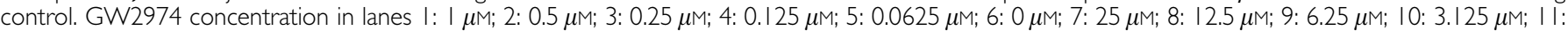
I.56 $\mu \mathrm{M}$ and 12: $0 \mu \mathrm{M}$.

by this compound at similar concentrations. Among potential downstream signalling components, phospho-Erk1/2 was not affected; however phospho-Akt, phospho-Stat3 and phospho-Shc were inhibited by GW2974 in a dose-dependent manner (Figure 1C). These data suggest that GW2974 affects Met activity, directly or indirectly, in H441 cells. In contrast, in H1666 cells, GW2974 inhibited phosphorylation of EGFR at submicromolar concentrations, as well as downstream signalling molecules including Akt, Erk1/2, and Stat3, consistent with its activity in the proliferation assay. Interestingly, there was little basal phosphorylation of EGFR at Y1068, or basal phosphorylation of Met at Y1234/1235 in H1666 cells, and the baseline phosphorylation of these receptors and downstream signalling molecules were only modestly inhibited by the drug (Figure 1C).

\section{Constitutive activation of Met, Her2 and Her3 in H441 cells is inhibited by GW2974}

As NSCLC cells express many RTKs that could potentially contribute to GW2974 sensitivity, we used RTK antibody capture arrays to assess the effects of the drug on activation of 42 different receptors in parallel (Figure 2A). Cells were serum starved and then incubated with EGF for $10 \mathrm{~min}$ in the presence or absence of GW2974. Interestingly, Met, EGFR, Her2, Her3 and Ret were basally activated in H441 cells, even under serum-deprivation conditions. Stimulation with EGF further activated EGFR and Ret, but not Met, Her2 or Her3 (Figure 2A). The activation of all these receptors was inhibited by treatment with GW2974 (Figure 2A). A lighter exposure of RTK array experiment is shown as Supplementary Figure S1. Hence, EGFR, Met, Her2 and Her3 are all activated in this cell line without exogenous EGF or HGF. These data indicate constitutive activation of Met, Her2 and Her3 in this cell line, and cross-talk among the ErbB family receptors and Met.

Immunoblot analysis of these extracts confirmed the RTK array data showing constitutive activation of EGFR and Met and inhibition by GW2974 (Figure 2B and C). Epidermal growth factor was able to further activate the tyrosine phosphorylation of ErbB receptors, and this effect is abrogated by the $2 \mathrm{~h}$ treatment with GW2974 (Bands around $175 \mathrm{kDa}$ correspond to ErbB receptors in Figure 2B). Detailed investigation of the effect of EGF treatment showed further phosphorylation of EGFR at Y845, but no further activation at Y1068 of EGFR and Met were observed (Figure 2C). Similarly, no further activation of Akt and Stat3 were observed with EGF treatment, whereas Shc and Erk1/2 were further activated by EGF treatment. Treatment of serum-deprived cells with GW2974 for only $2 \mathrm{~h}$ before EGF activation was sufficient to inhibit phosphorylation of EGFR and Met similar to 5-day continuous treatment with GW2974 in 10\% serum (Figure 1C). GW2974 also reduced downstream phosphorylation of Stat3, Shc and Akt, although only reducing the EGF-dependent phosphorylation of Erk1/2 to baseline basal levels. Overall, these data show that GW2974 inhibits the activation of downstream signalling induced by EGF treatment.

\section{GW2974 does not inhibit HGF-dependent activation of Met in the absence of ErbB family receptors}

The strong activation of members of both Met and ErbB families in H441 cells suggests two possible mechanisms for the inhibitory activity of GW2974 towards Met. The compound could work directly through an off-target inhibition of Met in these cells. Alternatively, ErbB receptors targeted by GW2974 may normally promote phosphorylation of Met in these cells. This would be consistent with recent studies suggesting receptor-level cross-talk between ErbB receptors and Met (Radaeva et al, 1999; Jo et al, 2000; Engelman et al, 2007).

To determine whether GW2974 directly inhibits the activation of Met, independent of its activity on EGFR and Her2, we incubated serum-starved H441 cells with the Met ligand, HGF. GW2974 inhibited phosphorylation of Met in the absence or presence of 
A

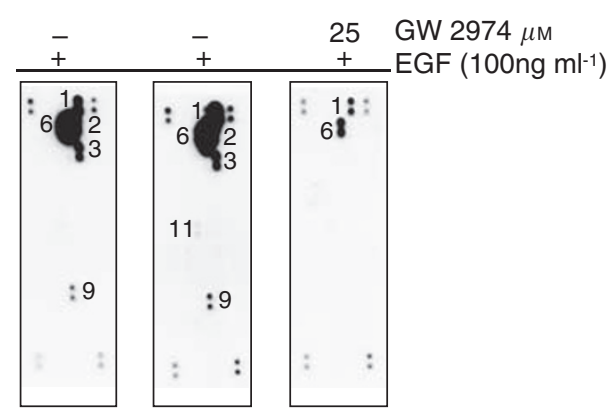

B

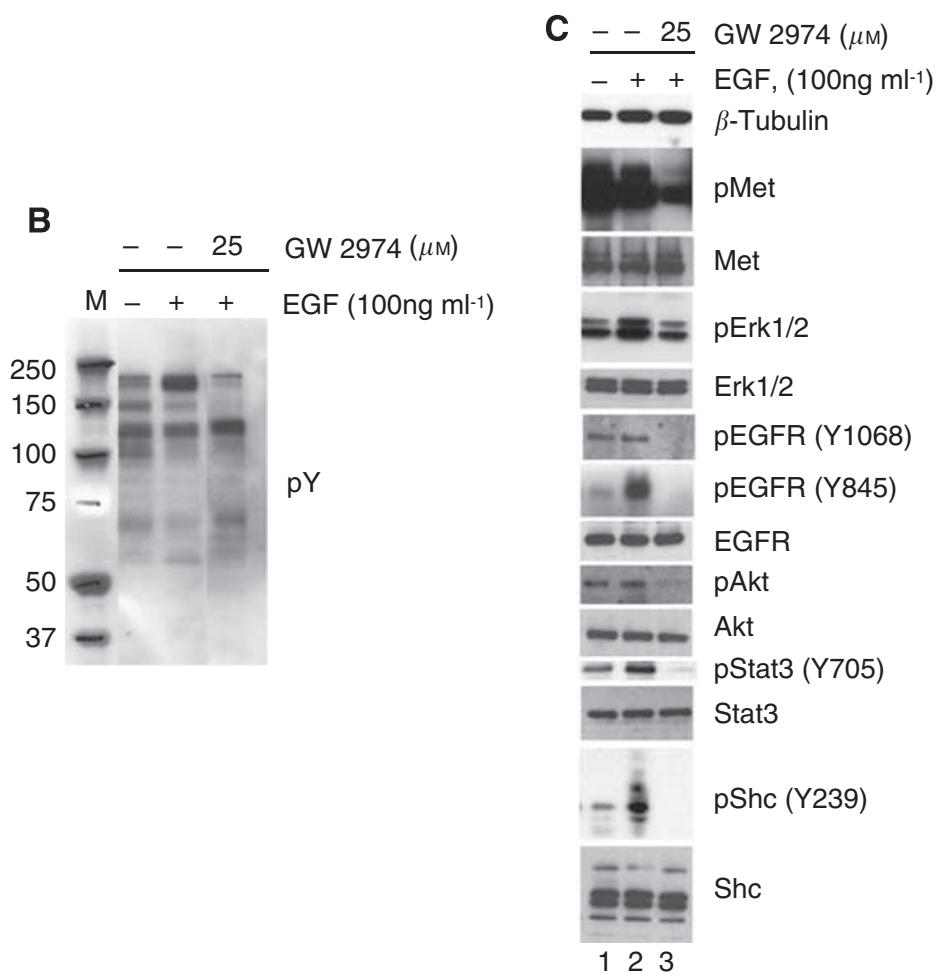

C

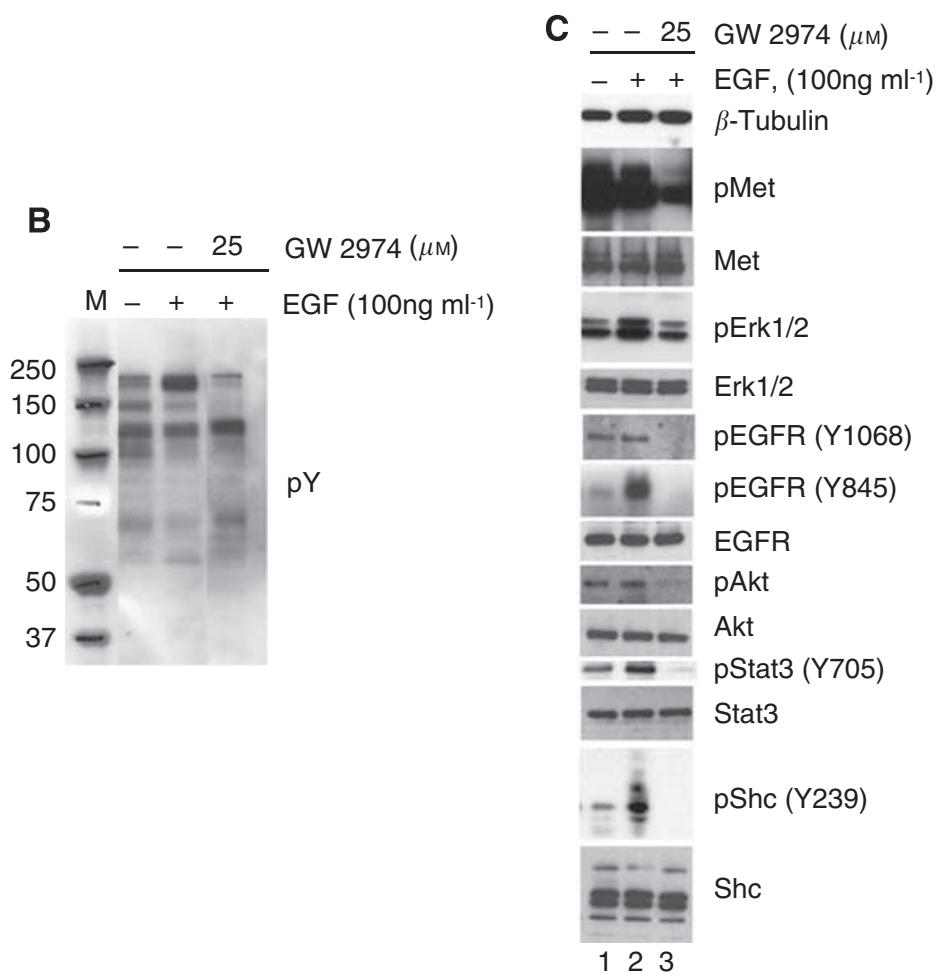

Figure 2 GW2974 inhibits constitutively activated Met, Her2 and Her3 in H44I cells. (A) GW2974 abrogates the activation of multiple receptors in $\mathrm{H} 44 \mathrm{I}$ cells. Cells were starved for $24 \mathrm{~h}$ and then treated for $2 \mathrm{~h}$ with GW2974 before incubation with EGF $\left(100 \mathrm{ng} \mathrm{ml} \mathrm{l}^{-1}\right.$ ) for $10 \mathrm{~min}$. Whole cell extracts $(500 \mu \mathrm{g})$ were analysed on each RTK array membrane, and activation status of receptors was assessed using antiphosphotyrosine antibody and numbered as in Figure IA. I: EGFR; 2: Her2; 3: Her3; 6: Met; 9: Ret; and I I: VEGFR-2. (B and C) GW2974 inhibits Met signalling and EGF-activated signalling. The extracts analysed in panel $A$ were analysed by immunoblotting with indicated antibodies. $M$ and numbers in panel $B$ indicate molecular weight marker for proteins. $\beta$-Tubulin was used as a loading control.

A

- $\quad$ - $2512.56 .253 .12-\quad$ - $2512.56 .253 .12 \mu \mathrm{M}$ GW2974

$-10--\quad-\quad-10-\quad-\quad \mu \mathrm{MPHA}$

$-{ }_{-}-{ }_{-}++++++\operatorname{HGF}\left(50 \mathrm{ng} \mathrm{ml}^{-1}\right)$

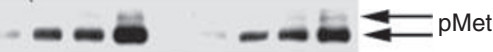

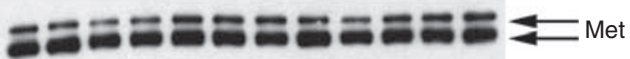

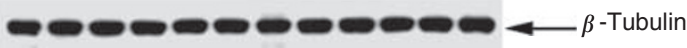

$\begin{array}{llllllllllll}1 & 2 & 3 & 4 & 5 & 6 & 7 & 8 & 9 & 10 & 11 & 12\end{array}$
B

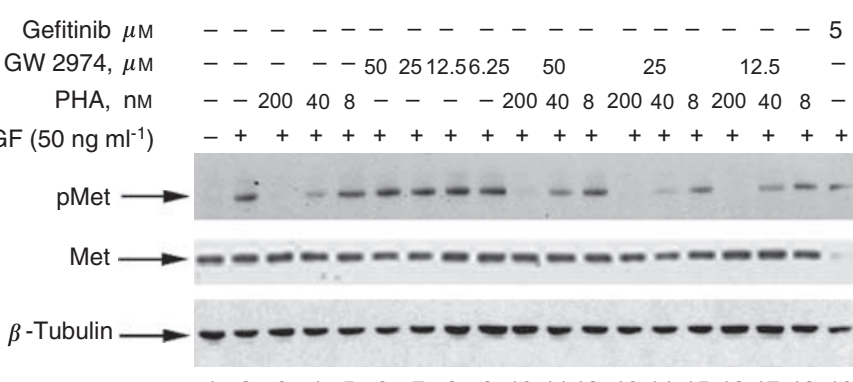

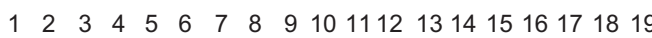

Figure 3 Cross-talk between Met and EGFR family receptors. GW2974 inhibits Met activation in a dose-dependent manner in H44I cells (A), but not in the absence of EGFR family receptors in 32D/Met cells (B). (A) GW2974 inhibits Met activation in the presence or absence of HGF. Cells were serum starved for $24 \mathrm{~h}$ followed by treatment with either vehicle (DMSO) or GW2974 or a Met inhibitor (PHA) for $2 \mathrm{~h}$ before activation with HGF (50 ng ml ${ }^{-1}$ ) for $30 \mathrm{~min}$. Whole cell extracts were made and subjected to western blot with indicated antibodies. (B) GW2974 and gefitinib do not inhibit HGF-dependent activation of Met in stably transfected 32D cells with Met (32D/Met). Cells were treated as in panel A. $\beta$-Tubulin was used as a loading control.

exogenous HGF in a dose-dependent manner similar to its activity in the presence of $10 \%$ foetal bovine serum (FBS) or EGF (Compare Figure $3 \mathrm{~A}$ with Figures 1 and 2). In addition, Met is constitutively active in H441 cells (Figures 1 and 2), and we did not observe any further phosphorylation in the presence of HGF (Figure 3A). Furthermore, the activity of GW2974 on Met phosphorylation was not influenced by the presence of HGF in these cells. A small molecule inhibitor of Met (PHA665752, referred to hereafter as PHA) was used as a positive control for Met inhibition.

To directly evaluate possible off-target effects of GW2974 on Met, we assayed activity on myeloid 32D cells stably transfected with Met (32D/Met) (Day et al, 1999). These cells do not express endogenous ErbB family receptors (EGFR, Her2, Her3 and Her4). GW2974 did not strongly inhibit the HGF-dependent activation of Met (Figure 3B, lanes 6-9), although there may be a marginal reduction in Met activation at the higher concentration of GW2974. Moreover, combination of the Met inhibitor, PHA, with GW2974 yielded similar activity to PHA alone, and further reduction in phosphorylation of Met was not observed (Figure 3B, lanes 10-18). Another small molecule EGFR inhibitor, gefitinib, did not inhibit Met activation in 32D/Met cells (Figure 3B, lane 19 and Supplementary Figure S2). These data suggest that, in H441 cells, the activity of GW2974 is due to the 
A

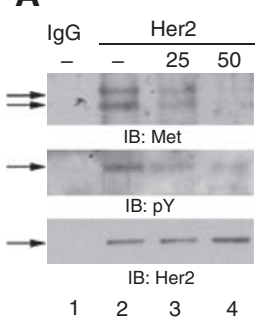

B

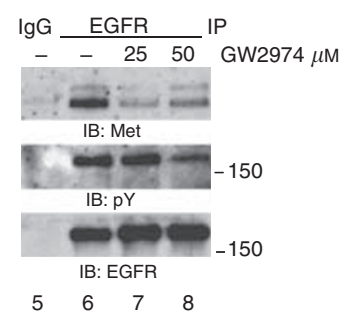

C

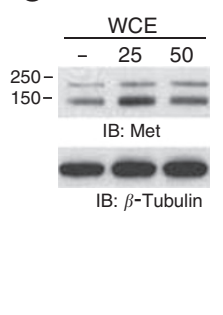

Figure 4 Met interacts with both EGFR and Her2, and the interaction is inhibited by GW2974. (A and B) Whole cell extracts were made from H44I cells treated with GW2974 for $2 \mathrm{~h}$ or DMSO vehicle control. Extracts (I mg) were subjected to immunoprecipitation with anti-Her2 (A) or with anti-EGFR (B) antibodies followed by immunoblotting with indicated antibodies. Immunoprecipitation with IgG was used as a negative control in each experiment. Numbers on the side of panels indicate molecular weight marker. The middle panels of A and B are from the ErbB receptor region of the blot. (C) Whole cell extracts were immunoblotted with anti-Met antibody to indicate equal amounts of Met in each treatment. $\beta$-Tubulin was used as a loading control.

inhibition of EGFR and/or Her2, which in turn affects the phosphorylation of Met. This suggests an active cross-talk between ErbB family receptors and Met.

\section{Physical interaction of Her2 and Met is inhibited by GW2974}

As GW2974 had little impact on Met in cells lacking ErbB family receptors, it is possible that, instead, the drug affects Met activation in H441 cells by inhibiting EGFR and/or Her2, and interfering secondarily with ErbB-dependent activation of Met. One possibility is that Met forms a physical complex with one or more ErbB family receptors. Indeed, Met coimmunoprecipitated with Her2, indicating that they are found in a protein complex (Figure 4A). This interaction was inhibited by GW2974 in a dosedependent fashion (compare lanes 3-4 with lane 2) paralleling reduction in Met phosphorylation (Figure 1C). Similarly, Met was coimmunoprecipitated with EGFR in H441 cells (Figure 4B). However, in this case, GW2974 reduced, but did not completely abrogate the interaction (compare lanes 7-8 with lanes 3-4). These data suggest that the cross-talk of EGFR and Her2, with Met is mediated through interactions in a protein complex, although the nature of the interactions with EGFR and Her2 may be different.

\section{Downregulation of Met affects the activation of ErbB family receptors}

It has been shown previously that the downregulation of Met, in a Met-overexpressing cell line with activating mutation in EGFR, did not reduce the activation of EGFR or Her3, but did restore gefitinib sensitivity (Engelman et al, 2007). We examined the effect of Met downregulation using Met-specific shRNA in H441 cells, which strongly reduced the expression of Met, but not EGFR, Her2 or Her3 (Figure 5B). In RTK capture array analysis, not only the Met phosphorylation but also the phosphorylation of ErbB family receptors (EGFR, Her2 and Her3) and Ret (Figure 5A) were reduced. Total tyrosine phosphorylation of immunoprecipitated Her2 and Her3 was reduced with the expression of Met shRNA (Figure 5C), consistent with the RTK capture array data (Figure 5A). Further analysis of the effects of Met shRNA on ErbB family receptors revealed that tyrosine phosphorylation sites in ErbB receptors are affected differentially. Both TK domain (Y847) as well as autophosphorylation sites (Y992, Y1068) of EGFR are affected more, with concurrent inhibition of Erk1/2 and Akt phosphorylation. In comparison, autophosphorylation sites of Her2 (Y1289, Y1112) and kinase domain (Y877) site were only modestly affected along with only marginal effect on Shc and no apparent effect on Stat3 phosphorylation status. Similarly, Her3 at Y1289 was less affected by the downregulation of Met (Figure 5B).
These data provide further evidence for the cross-talk of EGFR, Her2 and Her3 with Met.

\section{GW2974 inhibits wound healing in H441 cells}

As Met interacts with both EGFR and Her2, and Met-dependent signalling is inhibited by GW2974, we investigated the effects of GW2974 on a process associated with Met biological activity, wound healing. Earlier study showed that wound healing in H441 cells is inhibited by treatment with the Met inhibitor PHA (Christensen et al, 2003). GW2974 inhibited scratch wound healing of H441 cells at a concentration that inhibits other Met-dependent signalling processes (Supplementary Figure S3). The scratch wound in cells treated with GW2974 $(25 \mu \mathrm{M})$ was not healed by $54 \mathrm{~h}$ (Supplementary Figure $\mathrm{S} 3 \mathrm{H}$ ), whereas the scratch wound in untreated cells healed by $24 \mathrm{~h}$ in the presence of $10 \%$ FBS (Supplementary Figure S3C). Similarly, the scratch wound of cells expressing Met shRNA did not heal by $24 \mathrm{~h}$ in the presence of $10 \%$ FBS, whereas the scratch-wounded control cells were healed by $24 \mathrm{~h}$ (Supplementary Figure S4B and D ).

\section{Combination of inhibitors of Met with ErbB family inhibitors}

To explore the therapeutic implications of Met activation in the treatment of NSCLC, we combined the Met inhibitor with either gefitinib (Figure 6A) or GW2974 (Figure 6B) and monitored cell growth in H441 cells, H441-pLKO.1 and H441-Met shRNA cells. A fixed ratio of 1 was used for both PHA and GW2974 with concentrations ranging from 0.125 to $3 \mu \mathrm{M}$. Each data set is plotted as a dose-response regression curve. Each drug alone was unable to effectively inhibit the proliferation at concentrations used in this study, but the combination of two drugs resulted in a synergistic inhibition of proliferation. Importantly, the downregulation of Met restored both gefitinib and GW2974 activities (Figure 6Aiii and Biii). Western blot analysis indicates that GW2974 is more effective in inhibiting phosphorylation of Her2, Her3, Stat3 and Shc in H441-Met shRNA cells compared with control-infected H441pLKO.1 cells (Figure 6C) suggesting that Met is responsible for the activation of EGF family receptor-dependent signalling and resistance to GW2974 in H441 cells.

The combination of GW2974 with PHA yielded greater synergistic inhibition at lower concentration of both drugs than gefitinib with PHA for cell growth in H441-Met shRNA cells (compare Figure 6Aiii with Figure 6Biii), suggesting that Met directly affects the activation of EGFR in H441 cells. These data, when combined with other data presented in this study, suggest that simultaneous inhibition of multiple RTKs may be needed to effectively abrogate cell growth. 
A

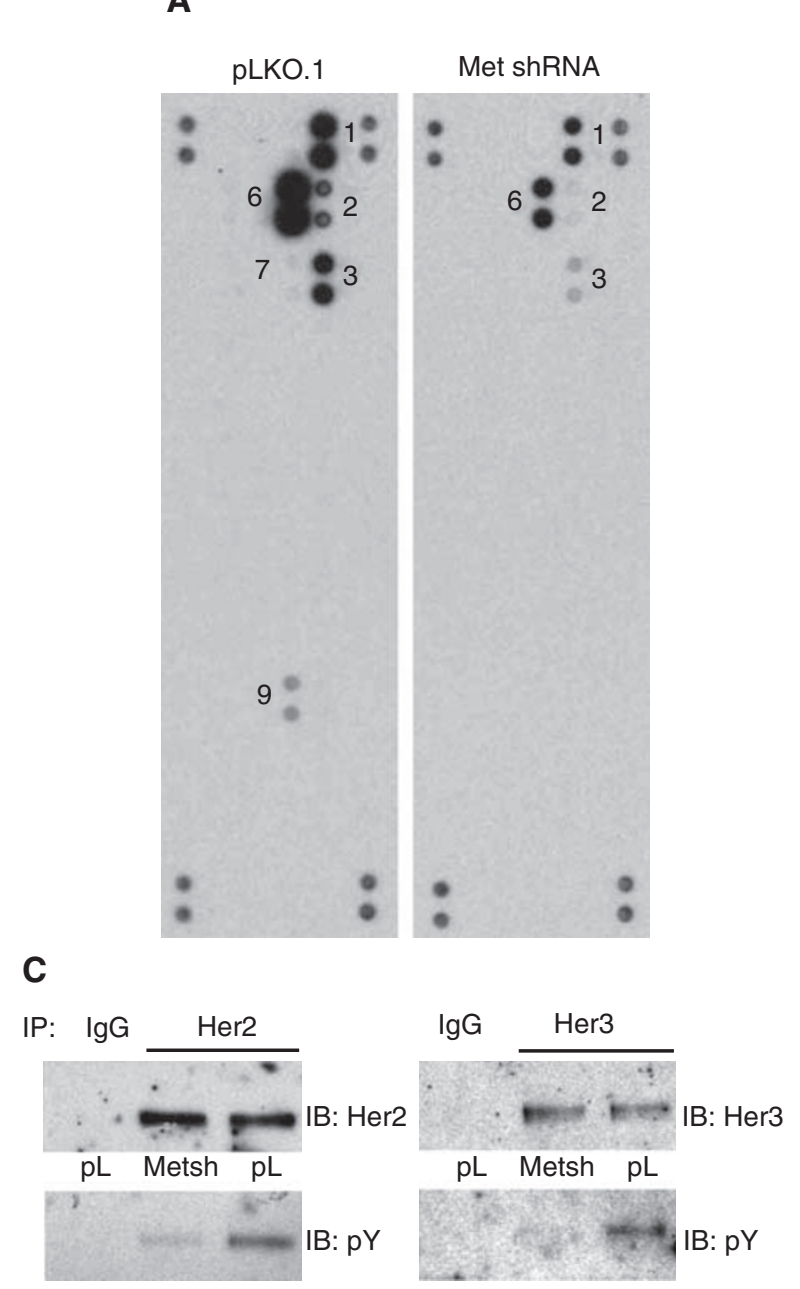

B

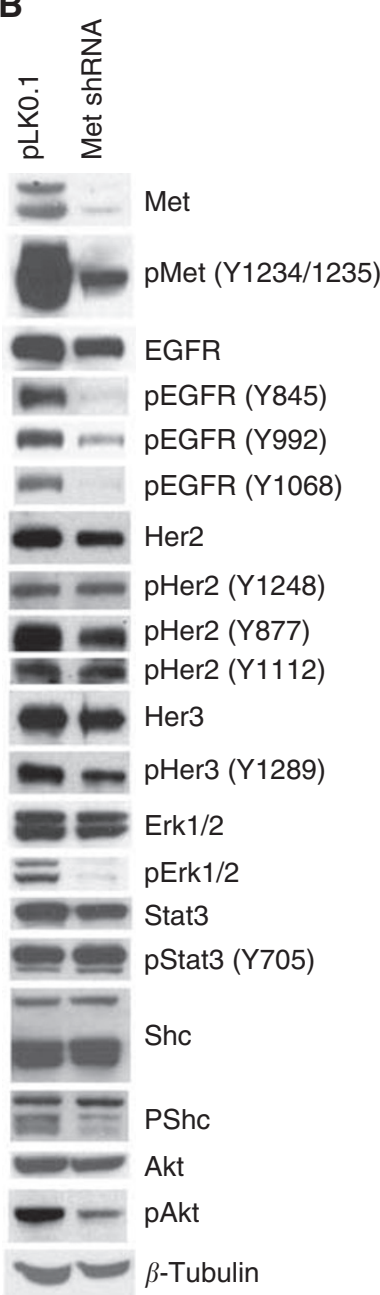

Figure 5 Met shRNA downregulates ErbB family receptor activation. Whole cell extracts were made from control (H44I-pLKO.I) and Met shRNA ( H44I-Met shRNA) cells. (A) Whole cell extracts $(200 \mu \mathrm{g})$ were analysed on each RTK array membrane, and activation status of receptors was assessed using antiphosphotyrosine antibody and numbered as in Figure IA. I: EGFR; 2: Her2; 3: Her3; 6: Met; 7: MSRP; and 9: Ret. (B) The cell extracts analysed in panel A were analysed by immunoblotting with indicated antibodies. $\beta$-Tubulin was used as a loading control. (C) Cell extracts (500 $\mu \mathrm{g})$ used in panel $\mathrm{A}$ were subjected to immunoprecipitation using either anti-Her2 or anti-Her3 antibodies followed by immunoblotting with indicated antibodies. IgG was used as a negative control.

\section{DISCUSSION}

In an effort to better understand potential druggable targets in NSCLC, we screened several cell lines with RTK capture arrays. These experiments revealed that, in H441 cells, the dominant RTK activity was for EGFR, Her2, Her3 and Met receptors. The H441 cell line has been previously shown to be resistant to gefitinib and cetuximab (Tracy et al, 2004; Mukohara et al, 2005). We found that the activity of EGFR in these cells can be inhibited by the dual EGFR and Her2 kinase inhibitor GW2974 at high micromolar concentrations, which also affects the phosphorylation of Stat3, Akt and Shc, but not Erk1/2. Unexpectedly, this inhibitor also reduced the high level of basal Met phosphorylation. The inhibition of Met by GW2974 seems to occur mainly by an indirect mechanism, as this compound has no effect on Met phosphorylation in Met-expressing 32D cells (32D/Met) that lack EGFR and Her2 receptors. As Met is coimmunoprecipitated with Her2 and EGFR, and this interaction is inhibited by GW2974, it is suggested that the disruption of physical complexes among RTKs is one possible mechanism. GW974 does have strong biological activities that probably work through Met, including the inhibition of wound healing after scratch injury of a cell monolayer. Moreover, combinations of anti-Met and anti-ErbB drugs yielded synergistic effects on cell proliferation and on downstream signalling from these receptors.

Activated EGFR, Her2, Her3 and Her4 form heterodimers that lead to cooperative and synergistic outputs. The close cooperativity of EGFR with Her2 has led to the development of other ErbB receptor family inhibitor drugs including the dual EGFR/HER2 TKI lapatinib and therapeutic antibody, pertuzumab, which blocks Her2 interactions with EGFR and Her3. These drugs are currently in clinical trials (pertuzumab) or approved (lapatinib), showing their promising activity in cancer. These receptors are also modulated by direct association with other receptors (including gp130), and by the impact of other receptors on functional activation of growth factor propeptides by proteolytic processing (e.g., Hb-EGF activation by G-protein-coupled receptors).

To date, there are no targeted therapies that are appropriate for the majority of NSCLC patients who do not respond to erlotinib or gefitinib. Presumably, these cancers are driven by the activation of other signalling molecules beyond the EGFR. Some will have mutations in other RTKs that would be hallmarks of sensitivity to 
A
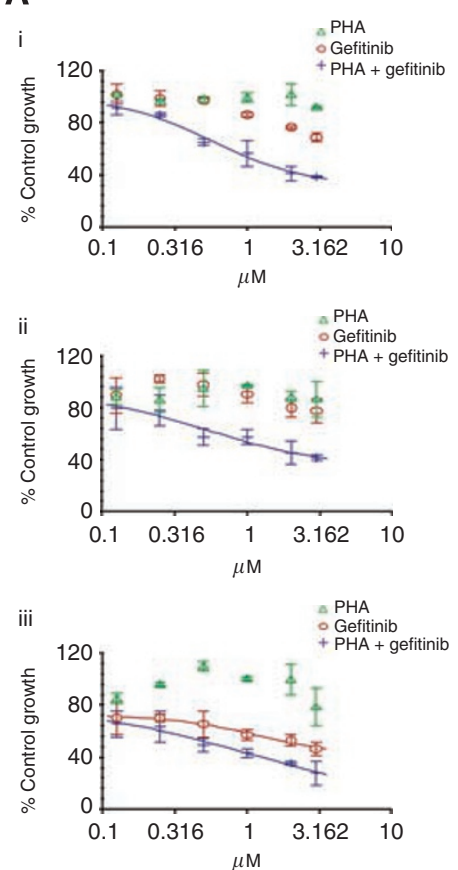

B
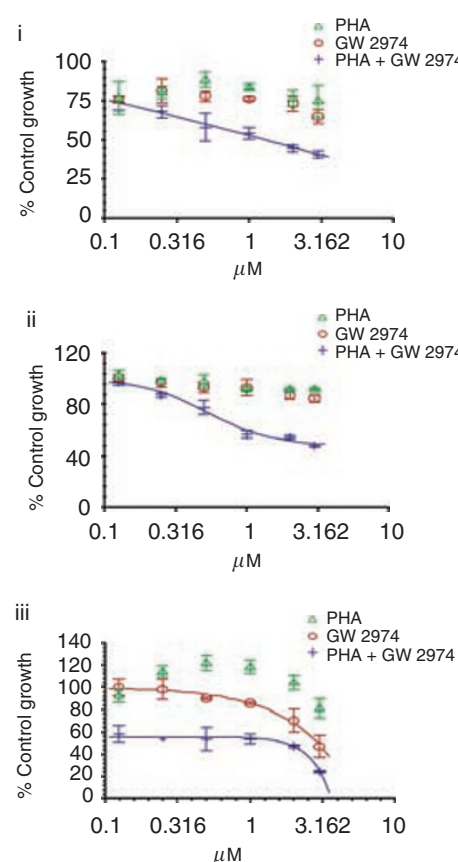

C

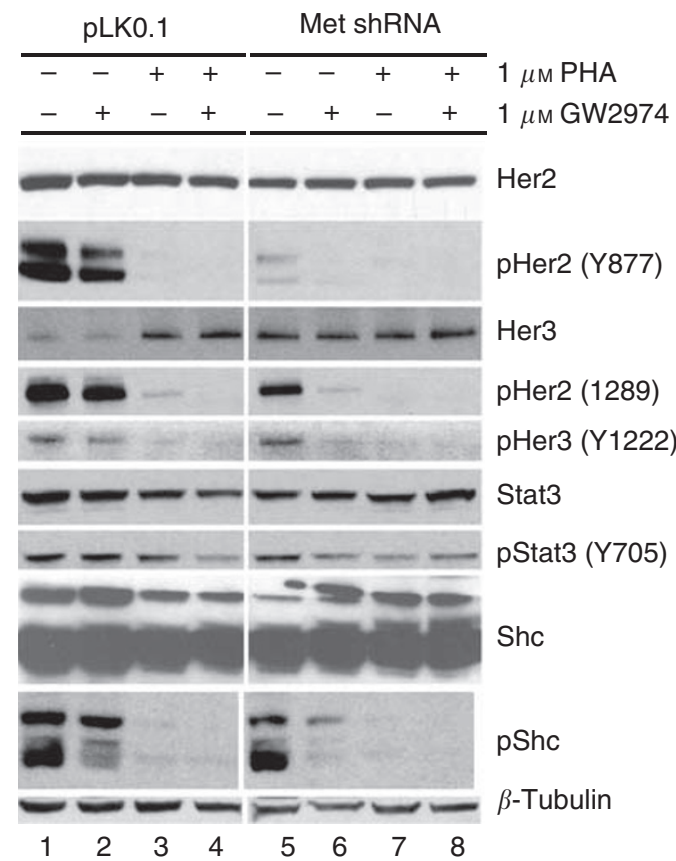

Figure 6 Synergistic inhibition of cell proliferation by combination of a Met inhibitor (PHA) with either gefitinib or GW2974 in H44I cells. Cells were treated with indicated concentrations of gefitinib and PHA (Ai-iii) or GW2974 and PHA (Bi-iii) either alone or in combination at $0.125-3 \mu \mathrm{M}$ concentration range for 5 days. For combination of drugs, a fixed ratio of I was used. Cell proliferation was assessed as in Figure IB. Data were plotted as dose-response regression curves using Xlfit program. Each error bar represents the s.d. from three data sets. Ai-Bi: H44I cells; $\mathbf{A i i}-\mathbf{B i i}$ : H44I-pLKO.I cells; and Aiii-Biii: H44I-Met shRNA cells. (C) Whole cell extracts were made from cells treated with drugs for 5 days as indicated, and were subjected to western blot analysis with indicated antibodies. $\beta$-tubulin was used as a loading control.

RTK inhibitors with the appropriate action spectrum, whereas others may be driven by mechanisms unrelated to RTKs. Profiling tumours with RTK capture arrays may be very helpful in first-pass analysis of tumours to identify active RTKs that may be susceptible to treatment with the kinase inhibitors. For example, our finding that H441 cells are sensitive to Met, and ErbB inhibitors could be predicted based on the activity of these receptors determined by RTK capture array (Figure 1).

Besides posing treatment challenges, the cross-interactivity of RTKs potentially provides new treatment opportunities. This study is focused on the interactions between two RTK families, ErbBs and Met, that have overlapping functions in initiation and progression of cancer. ErbBs are implicated in promoting proliferation and survival, and Met has important roles in cell motility and epithelial/mesenchymal transition. Our identification of ErbB and Met signalling in a single lung cancer cell line, and the involvement in responsiveness to $\mathrm{ErbB}$ and Met inhibitors is consistent with other recent studies that highlighted functional cooperation between these two receptor families (Bean et al, 2007; Guo et al, 2008; Mueller et al, 2008; Shattuck et al, 2008; Yano et al, 2008). Interaction of these receptor families has been suggested for years, based on the finding that Met is often co-overexpressed with ErbBs in prostate and breast carcinoma. Hepatocyte growth factor promotes motility and invasive behaviour by Her2 (Neu)transformed mammary epithelial cells (Khoury et al, 2005). A recent phosphoproteomic screen for EGFR targets in glioblastoma identified Met (Stommel et al, 2007).

Receptor tyrosine kinase antibody arrays used in this study clearly indicated differences in receptor activation levels in two cell lines with WT EGFR, which prompted us to investigate ErbB family receptor inhibitors in NSCLC. In this study, we report the activity of a lapatinib analogue, GW2974, which is a small molecule dual TKI of EGFR/Her2. Lapatinib has shown preferential benefit for Her2 amplified breast cancer patients compared with patients with normal level of Her2 (Konecny et al, 2006). Although HER2 is not commonly amplified in NSCLC, in approximately $2 \%$ of NSCLC patients, an activating mutation similar to those observed in EGFR has been reported in Her2. The response rate for lapatinib alone in a randomised clinical trial for NSCLC was very low (Smylie et al, 2007). We have shown that GW2974 does show a high micromolar activity in H441 cells that carry WT EGFR and constitutive activation and expression of Met. This activity is similar to non-amplified Her2 in breast cancer (Konecny et al, 2006). However, we report a GW2974-sensitive lung cancer cell line (H1666) that has normal level of both Her2 and Met, and does not have activating mutation in EGFR. GW2974 was able to downregulate EGFR-dependent activation of Akt, Erk1/2, and had a marginal effect on Stat 3 in a dose-dependent fashion (Figure 1C). This cell line has been shown to be resistant to gefitinib. Sensitivity of this cell line to GW2974 is similar to its activity in Her2 amplified breast cancers. Further investigation is necessary to understand the molecular mechanism for its response to this drug.

On the contrary, GW2974 downregulated Met-dependent signalling in H441 and showed no effect on the phosphorylation level of Erk1/2. Constitutive activation of Met, Her2 and Her3 even in serum-starved cells suggests a ligand-independent activation of these receptors. One possibility may be autocrine activation of these receptors due to the expression of a ligand, such as EGF or HGF etc. The expression of ligand by cancer cells has been observed in many cases that often lead to drug resistance bypassing a need for the overexpression or activating mutation in oncogenes for cancer progression. A second possibility is the transactivation by another receptor, for example, Met in H441 cells that bypasses the need for ligand-dependent activation of EGFR, Her2 and Her3 (Figures 1, 2, 5 and 6). GW2974 inhibited the proliferation, Met-dependent activation of downstream signalling 
and phosphorylation of Met in a dose-dependent manner in H441 cells, but did not downregulate the HGF-dependent activation of Met in 32D/Met cells suggesting that in H441, there is an active cross-talk between Met and ErbB receptors. However, the inhibitory activity of GW2974 is observed at higher (micromolar) concentration than is required normally for the inhibition of EGFR/Her2-dependent signalling. In H441 cells, GW2974 only inhibited the activation of EGFR, Her2 and Her3 at lower concentrations, when Met was downregulated (Figure 6, compare lane 2 with lane 6). Indeed, both Her2 and EGFR are able to coimmunoprecipitate Met in H441 cells. The interaction of Met with Her2 is inhibited by GW2974 in a dose-dependent fashion similar to its activity towards Met-dependent signalling. However, the interaction of Met with EGFR is of a different nature, as this interaction is inhibited by GW2974 without showing any dose dependence. For the first time, we have shown that the downregulation of Met by Met-specific shRNA leads to differential effect on the tyrosine phosphorylation sites of EGFR, Her2 and Her3 (Figure 5) suggesting that Met plays a crucial role in activating ErbB family receptors especially EGFR, as the activation of EGFR is drastically affected with concomitant inhibition of Erk1/2 and Akt activation. However, activation of Stat 3 was not affected, and Shc is only marginally affected by Met downregulation suggesting that it is regulated by different mechanism. One such mechanism could be ligand-dependent activation of Her2 and Her3 in these cells, as the activation of both are downregulated by GW2974 in a dosedependent manner.

To explore the therapeutic implications of coactivation of RTKs, we evaluated the combination of inhibitors for Met and ErbB family receptors in H441 cells. As shown in Figure 6, either drug alone was not very effective at inhibiting proliferation. However, the combination of drugs was synergistic in inhibiting the proliferation of cells very effectively. Interestingly, it is very clear from regression curves that any small inhibition by PHA alone was sufficient to effectively inhibit the cell growth when combined with GW2974 at the lowest concentration of $0.125 \mu \mathrm{M}$ in Met shRNA cells (Figure 6Biii), whereas this was not the case with PHA and gefitinib in the same cells (Figure 6Aiii). This suggests that the coactivation of multiple RTKs in cancer cells confer resistance to single-agent therapy, but this resistance can easily be overcome by simultaneously inhibiting several RTKs with rationally chosen drug combinations. Similar observations were made recently in the case of glioblastomas carrying activated EGFR and Met receptors where simultaneous inhibition of EGFR (erlotinib) and Met (SU11274) lead to the downregulation of PI3K pathway, and reduced the size of colony formation in soft agar (Stommel et al, 2007). The single inhibition by single drug was less effective. Thus, our study combined with other similar observations provides a compelling evidence to effectively treat cancers by rationally designed drug combinations.

The rapid pace of development of molecularly targeted anticancer drugs creates a concomitant need for the development of the best tools for matching the drugs to appropriate patients. Phosphoproteomic analysis by RTK capture arrays may be a valuable tool for identifying the subset of tumours with functional receptor activation, regardless of mechanism. Similar arrays that monitor the activity of intermediary signalling molecules through their phosphorylation status provide one relatively inexpensive tool for this purpose. The best analysis of tumour samples may require a combination of proteomic, genetic, epigenetic and functional characterisation.

The functional studies here led to the hypothesis that dual targeting of ErbBs and Met may be very important in the treatment of some human cancers. Ectopic signalling by members of each receptor family are common in human cancer, and the receptor systems have complementary functions in promoting growth, survival and qualities associated with invasion and metastasis. This study when combined with other studies provides compelling support for the combination of these drugs in future clinical trials.

\section{ACKNOWLEDGEMENTS}

We are thankful to Dr Donald P Bottaro from the NCI, NIH (Bethesda, USA) for providing us with 32D/Met cell line, and to Dr JA Engelman (MGH Cancer Center, Boston, USA) for providing us with shRNA construct for Met. We are also thankful to Jennifer Bordeaux (Yale University) for technical assistance in the preparation of this study. This study was supported by The Ethel F Donaghue Women's Health Research Program at Yale to MPD; the Yale PREP Program for JK, R33 Grant NIH CA 110511 to DLR and NIH Public Health Service Grant R01 CA45708 to DFS.

Supplementary Information accompanies the paper on British Journal of Cancer website (http://www.nature.com/bjc)

\section{REFERENCES}

Arteaga CL (2007) HER3 and mutant EGFR meet MET. Nat Med 13: $675-677$

Balak MN, Gong Y, Riely GJ, Somwar R, Li AR, Zakowski MF, Chiang A, Yang G, Ouerfelli O, Kris MG, Ladanyi M, Miller VA, Pao W (2006) Novel D761Y and common secondary T790M mutations in epidermal growth factor receptor-mutant lung adenocarcinomas with acquired resistance to kinase inhibitors. Clin Cancer Res 12: 6494-6501

Bean J, Brennan C, Shih JY, Riely G, Viale A, Wang L, Chitale D, Motoi N, Szoke J, Broderick S, Balak M, Chang WC, Yu CJ, Gazdar A, Pass H, Rusch V, Gerald W, Huang SF, Yang PC, Miller V, Ladanyi M, Yang CH, Pao W (2007) MET amplification occurs with or without T790M mutations in EGFR mutant lung tumors with acquired resistance to gefitinib or erlotinib. Proc Natl Acad Sci USA 104: 20932-20937

Christensen JG, Schreck R, Burrows J, Kuruganti P, Chan E, Le P, Chen J, Wang X, Ruslim L, Blake R, Lipson KE, Ramphal J, Do S, Cui JJ, Cherrington JM, Mendel DB (2003) A selective small molecule inhibitor of c-Met kinase inhibits c-Met-dependent phenotypes in vitro and exhibits cytoreductive antitumor activity in vivo. Cancer Res 63: 7345-7355

Day RM, Cioce V, Breckenridge D, Castagnino P, Bottaro DP (1999) Differential signaling by alternative HGF isoforms through c-Met: activation of both MAP kinase and PI 3-kinase pathways is insufficient for mitogenesis. Oncogene 18: 3399-3406
Desiderio MA (2007) Hepatocyte growth factor in invasive growth of carcinomas. Cell Mol Life Sci 64: 1341-1354

Engelman JA, Janne PA, Mermel C, Pearlberg J, Mukohara T, Fleet C, Cichowski K, Johnson BE, Cantley LC (2005) ErbB-3 mediates phosphoinositide 3-kinase activity in gefitinib-sensitive non-small cell lung cancer cell lines. Proc Natl Acad Sci USA 102: 3788 - 3793

Engelman JA, Zejnullahu K, Mitsudomi T, Song Y, Hyland C, Park JO, Lindeman N, Gale CM, Zhao X, Christensen J, Kosaka T, Holmes AJ, Rogers AM, Cappuzzo F, Mok T, Lee C, Johnson BE, Cantley LC, Janne PA (2007) MET amplification leads to gefitinib resistance in lung cancer by activating ERBB3 signaling. Science 316: 1039-1043

Gao CF, Vande Woude GF (2005) HGF/SF-Met signaling in tumor progression. Cell Res 15: 49-51

Gao CF, Xie Q, Su YL, Koeman J, Khoo SK, Gustafson M, Knudsen BS, Hay R, Shinomiya N, Vande Woude GF (2005) Proliferation and invasion: plasticity in tumor cells. Proc Natl Acad Sci USA 102: $10528-10533$

Guo A, Villen J, Kornhauser J, Lee KA, Stokes MP, Rikova K, Possemato A, Nardone J, Innocenti G, Wetzel R, Wang Y, MacNeill J, Mitchell J, Gygi SP, Rush J, Polakiewicz RD, Comb MJ (2008) Signaling networks assembled by oncogenic EGFR and c-Met. Proc Natl Acad Sci USA 105: $692-697$ 
Haber DA, Bell DW, Sordella R, Kwak EL, Godin-Heymann N, Sharma SV, Lynch TJ, Settleman J (2005) Molecular targeted therapy of lung cancer: EGFR mutations and response to EGFR inhibitors. Cold Spring Harb Symp Quant Biol 70: 419-426

Jo M, Stolz DB, Esplen JE, Dorko K, Michalopoulos GK, Strom SC (2000) Cross-talk between epidermal growth factor receptor and c-Met signal pathways in transformed cells. J Biol Chem 275: 8806-8811

Khoury H, Naujokas MA, Zuo D, Sangwan V, Frigault MM, Petkiewicz S, Dankort DL, Muller WJ, Park M (2005) HGF converts ErbB2/Neu epithelial morphogenesis to cell invasion. Mol Biol Cell 16: $550-561$

Konecny GE, Pegram MD, Venkatesan N, Finn R, Yang G, Rahmeh M, Untch M, Rusnak DW, Spehar G, Mullin RJ, Keith BR, Gilmer TM, Berger M, Podratz KC, Slamon DJ (2006) Activity of the dual kinase inhibitor lapatinib (GW572016) against HER-2-overexpressing and trastuzumab-treated breast cancer cells. Cancer Res 66: 1630-1639

Kong-Beltran M, Seshagiri S, Zha J, Zhu W, Bhawe K, Mendoza N, Holcomb T, Pujara K, Stinson J, Fu L, Severin C, Rangell L, Schwall R, Amler L, Wickramasinghe D, Yauch R (2006) Somatic mutations lead to an oncogenic deletion of met in lung cancer. Cancer Res 66: 283-289

Lengyel E, Prechtel D, Resau JH, Gauger K, Welk A, Lindemann K, Salanti G, Richter T, Knudsen B, Vande Woude GF, Harbeck N (2005) C-Met overexpression in node-positive breast cancer identifies patients with poor clinical outcome independent of Her2/neu. Int $J$ Cancer 113: $678-682$

Liu B, Bernard B, Wu JH (2006) Impact of EGFR point mutations on the sensitivity to gefitinib: insights from comparative structural analyses and molecular dynamics simulations. Proteins 65: 331-346

Ma PC, Maulik G, Christensen J, Salgia R (2003) c-Met: structure, functions and potential for therapeutic inhibition. Cancer Metastasis Rev 22: $309-325$

Mueller KL, Hunter LA, Ethier SP, Boerner JL (2008) Met and c-Src cooperate to compensate for loss of epidermal growth factor receptor kinase activity in breast cancer cells. Cancer Res 68: 3314-3322

Mukohara T, Engelman JA, Hanna NH, Yeap BY, Kobayashi S, Lindeman N, Halmos B, Pearlberg J, Tsuchihashi Z, Cantley LC, Tenen DG, Johnson BE, Janne PA (2005) Differential effects of gefitinib and cetuximab on non-small-cell lung cancers bearing epidermal growth factor receptor mutations. J Natl Cancer Inst 97: 1185-1194

Petrov KG, Zhang YM, Carter M, Cockerill GS, Dickerson S, Gauthier CA, Guo Y, Mook Jr RA, Rusnak DW, Walker AL, Wood ER, Lackey KE (2006) Optimization and SAR for dual ErbB-1/ErbB-2 tyrosine kinase inhibition in the 6-furanylquinazoline series. Bioorg Med Chem Lett 16: $4686-4691$

Pozner-Moulis S, Pappas DJ, Rimm DL (2006) Met, the hepatocyte growth factor receptor, localizes to the nucleus in cells at low density. Cancer Res 66: $7976-7982$
Radaeva S, Ferreira-Gonzalez A, Sirica AE (1999) Overexpression of C-NEU and C-MET during rat liver cholangiocarcinogenesis: A link between biliary intestinal metaplasia and mucin-producing cholangiocarcinoma. Hepatology 29: 1453-1462

Riely GJ, Politi KA, Miller VA, Pao W (2006) Update on epidermal growth factor receptor mutations in non-small cell lung cancer. Clin Cancer Res 12: $7232-7241$

Rosario M, Birchmeier W (2003) How to make tubes: signaling by the Met receptor tyrosine kinase. Trends Cell Biol 13: 328-335

Rusnak DW, Affleck K, Cockerill SG, Stubberfield C, Harris R, Page M, Smith KJ, Guntrip SB, Carter MC, Shaw RJ, Jowett A, Stables J, Topley P, Wood ER, Brignola PS, Kadwell SH, Reep BR, Mullin RJ, Alligood KJ, Keith BR, Crosby RM, Murray DM, Knight WB, Gilmer TM, Lackey K (2001) The characterization of novel, dual ErbB-2/EGFR, tyrosine kinase inhibitors: potential therapy for cancer. Cancer Res 61: 7196-7203

Sattler M, Salgia R (2007) c-Met and hepatocyte growth factor: potential as novel targets in cancer therapy. Curr Oncol Rep 9: 102-108

Sharma SV, Bell DW, Settleman J, Haber DA (2007) Epidermal growth factor receptor mutations in lung cancer. Nat Rev Cancer 7: 169-181

Shattuck DL, Miller JK, Carraway III KL, Sweeney C (2008) Met receptor contributes to trastuzumab resistance of Her2-overexpressing breast cancer cells. Cancer Res 68: 1471 - 1477

Smylie M, Blumenschein GR, Dowlati A, Garst J, Shepherd FA, Rigas JR, Hassani H, Berger MS, Zaks T, Ross HJ (2007) A phase II multicenter trial comparing two schedules of lapatinib (LAP) as first or second line monotherapy in subjects with advanced or metastatic non-small cell lung cancer (NSCLC) with either bronchioloalveolar carcinoma (BAC) or no smoking history. J Clin Oncol 25: 7611 (2007 Asco Annual Meeting)

Sordella R, Bell DW, Haber DA, Settleman J (2004) Gefitinib-sensitizing EGFR mutations in lung cancer activate anti-apoptotic pathways. Science 305: $1163-1167$

Stommel JM, Kimmelman AC, Ying H, Nabioullin R, Ponugoti AH, Wiedemeyer R, Stegh AH, Bradner JE, Ligon KL, Brennan C, Chin L, DePinho RA (2007) Coactivation of receptor tyrosine kinases affects the response of tumor cells to targeted therapies. Science 318: 287-290

Tracy S, Mukohara T, Hansen M, Meyerson M, Johnson BE, Janne PA (2004) Gefitinib induces apoptosis in the EGFRL858R non-small-cell lung cancer cell line H3255. Cancer Res 64: $7241-7244$

Yang Y, Spitzer E, Meyer D, Sachs M, Niemann C, Hartmann G, Weidner KM, Birchmeier C, Birchmeier W (1995) Sequential requirement of hepatocyte growth factor and neuregulin in the morphogenesis and differentiation of the mammary gland. J Cell Biol 131: 215-226

Yano S, Wang W, Li Q, Matsumoto K, Sakurama H, Nakamura T, Ogino H, Kakiuchi S, Hanibuchi M, Nishioka Y, Uehara H, Mitsudomi T, Yatabe Y, Nakamura T, Sone S (2008) Hepatocyte growth factor induces gefitinib resistance of lung adenocarcinoma with epidermal growth factor receptor-activating mutations. Cancer Res 68: 9479-9487 